\title{
NMR-Detected Host-Guest Proton Exchange as a Tool to Explore Surface/Volume Ratios and Fluid Filling of Internal and External Spaces of Porous Solids containing Surface-OH-Groups
}

Verónica Torres-Barthelemy, ${ }^{a}$ Natalia Pérez-Hernández, ${ }^{b}$ Ilya G. Shenderovich, ${ }^{c}$ Peter M. Tolstoy, ${ }^{d}$

Gleb S. Denisov, ${ }^{e}$ and Hans-Heinrich Limbach ${ }^{f *}$

Corresponding Author

Hans-Heinrich Limbach -Institut für Chemie und Biochemie, Freie Universität Berlin, Takustrasse 3, 14195 Berlin, Germany; orcid.org/0000-0002-2084-6359; Email: limbach@chemie.fu-berlin.de

Authors

${ }^{a}$ Institut für Chemie und Biochemie, Freie Universität Berlin, Takustrasse 3, 14195 Berlin, Germany; Present Address: Bayer Pharma AG, Berlin, Email: vtorresbarthelemy@gmail.com

${ }^{b}$ Institut für Chemie und Biochemie, Freie Universität Berlin, Takustrasse 3, 14195 Berlin, Germany and Institute for Chemical Research (CSIC-US), C/Américo Vespucio 49, E-41092 Seville, Spain. Present Address: National Institute for Toxicology and Forensic Science, Sevilla, Spain. Email: nataliaperezh@outlook.es

${ }^{c}$ Faculty of Chemistry and Pharmacy, University of Regensburg, Universitaetstrasse 31, 93053 Regensburg, Germany; orcid.org/0000-0001-6713-9080. Email: Ilya.Shenderovich@chemie.uniregensburg.de

${ }^{d}$ St. Petersburg State University, Universitetskij pr. 26, 198504, St. Petersburg, Russian Federation; orcid.org/0000-0002-8426-3988. Email: peter.tolstoy@spbu.ru

${ }^{e}$ Department of Physics, St. Petersburg State University, Ulyanovskaya st.1, 198504 St. Petersburg, Russian Federation; orcid.org/0000-0002-1246-0552. Email: gldenisov@yandex.ru

${ }^{f}$ Institut für Chemie und Biochemie, Freie Universität Berlin, Takustrasse 3, 14195 Berlin, Germany

\section{Supplementary Information}

\section{Sample Composition}

The samples were initially characterized by the mass ratio $W=m_{\mathrm{MPz}} / m_{\text {silica }}$, where $m_{\mathrm{MPz}}$ represents the mass in mg of 4-methylpyrazole (MPz) and $m_{\text {silica }}$ the mass in mg of the silica used, MCM-41 or SBA-15. The molar ratios of the NH groups of MPz and of the SiOH groups of silica, $R=N_{\mathrm{NH}} / N_{\mathrm{OH}}$, were determined by ${ }^{1} \mathrm{H}$ MAS NMR as described in the main text. $X_{\mathrm{NH}}$ represents the mole fraction of the NH groups of MPz. 
Table S1. Composition of 4-methylpyrazole (MPz) inside silica of the MCM-41 type and ${ }^{1} \mathrm{H}$ MAS Chemical Shifts $\delta$ at $398 \mathrm{~K}$

\begin{tabular}{|c|c|c|c|}
\hline$W=m_{\mathrm{MPz}} / m_{\text {silica }}$ & $R=N_{\mathrm{NH}} / N_{\mathrm{OH}}$ & $X_{\mathrm{NH}}=R /(1+R)$ & $\delta / \mathrm{ppm}$ \\
\hline 0.000 & 0.00 & 0.00 & 1.7 \\
\hline 0.006 & 0.01 & 0.01 & 1.9 \\
\hline 0.010 & 0.05 & 0.05 & 3.3 \\
\hline 0.014 & 0.02 & 0.02 & 2.7 \\
\hline 0.019 & 0.04 & 0.04 & 3.2 \\
\hline 0.020 & 0.08 & 0.07 & 3.9 \\
\hline 0.029 & 0.10 & 0.09 & 4.1 \\
\hline 0.039 & 0.18 & 0.15 & 4.8 \\
\hline 0.049 & 0.19 & 0.16 & 5.4 \\
\hline 0.058 & 0.18 & 0.15 & 5.6 \\
\hline 0.066 & 0.32 & 0.24 & 6.7 \\
\hline 0.078 & 0.22 & 0.18 & 6.1 \\
\hline 0.085 & 0.30 & 0.23 & 6.9 \\
\hline 0.097 & 0.25 & 0.20 & 6.7 \\
\hline 0.114 & 0.24 & 0.19 & 7.1 \\
\hline 0.131 & 0.37 & 0.27 & 6.9 \\
\hline 0.203 & 0.48 & 0.32 & 8.0 \\
\hline 0.211 & 0.41 & 0.29 & 7.8 \\
\hline 0.221 & 0.50 & 0.33 & 8.3 \\
\hline 0.240 & 0.41 & 0.29 & 8.2 \\
\hline 0.264 & 0.61 & 0.38 & 8.2 \\
\hline 0.293 & 0.60 & 0.37 & 7.8 \\
\hline 0.335 & 0.54 & 0.35 & 8.5 \\
\hline 0.379 & 0.68 & 0.40 & 9.0 \\
\hline 0.427 & 1.02 & 0.50 & 9.5 \\
\hline \multirow[t]{2}{*}{0.446} & 1.28 & 0.56 & 10.1 \\
\hline & & & 9.6 \\
\hline \multirow[t]{3}{*}{1.28} & 2.75 & 0.73 & 11.7 \\
\hline & & & 11.8 \\
\hline & & & 9.7 \\
\hline \multirow[t]{2}{*}{4.18} & 8.13 & 0.89 & 11.9 \\
\hline & & & 12.0 \\
\hline$W=m_{\mathrm{MPz}} / m_{\text {silica }}$ & $R=N_{\mathrm{NH}} / N_{\mathrm{OH}}$ & $X_{\mathrm{NH}}=R /(1+R)$ & $\delta / \mathrm{ppm}$ \\
\hline
\end{tabular}


Table S2. Composition of 4-methylpyrazole (MPz) inside silica of the SBA-15 type and ${ }^{1}$ H MAS Chemical Shifts $\delta$ at 398 K

$\begin{array}{llll}W=m_{\mathrm{MPz}} / m_{\text {silica }} & R=N_{\mathrm{NH}} / N_{\mathrm{OH}} & X_{\mathrm{NH}}=R /(1+R) & \delta / \mathrm{ppm} \\ 0.000 & 0.00 & 0.00 & 1.7 \\ 0.009 & 0.01 & 0.01 & 2.0 \\ 0.014 & 0.06 & 0.06 & 2.7 \\ 0.023 & 0.04 & 0.04 & 4.1 \\ 0.028 & 0.06 & 0.06 & 3.7 \\ 0.042 & 0.07 & 0.07 & 3.9 \\ 0.056 & 0.08 & 0.07 & 4.5 \\ 0.057 & 0.07 & 0.07 & 4.6 \\ 0.070 & 0.13 & 0.12 & 4.9 \\ 0.074 & 0.08 & 0.07 & 5.0 \\ 0.085 & 0.09 & 0.08 & 5.1 \\ 0.099 & 0.12 & 0.11 & 5.5 \\ 0.113 & 0.18 & 0.15 & 5.8 \\ 0.127 & 0.19 & 0.16 & 6.1 \\ 0.148 & 0.17 & 0.15 & 6.1 \\ 0.170 & 0.33 & 0.25 & 6.5 \\ 0.183 & 0.35 & 0.26 & 7.5 \\ 0.268 & 0.34 & 0.25 & 6.9 \\ 0.282 & 0.38 & 0.28 & 7.9 \\ 1.26 & 1.55 & & 10.4 \\ & & 0.61 & 11.0 \\ 2.83 & 3.50 & & 10.4 \\ & & 0.77 & 11.3 \\ & & & \end{array}$


Figure S1. Selected single $90^{\circ}$ pulse ${ }^{1} \mathrm{H}$ MAS NMR spectra at $398 \mathrm{~K}$ and $7 \mathrm{~T}$ of 4-methyl- $1 \mathrm{H}$ pyrazole in MCM-41.

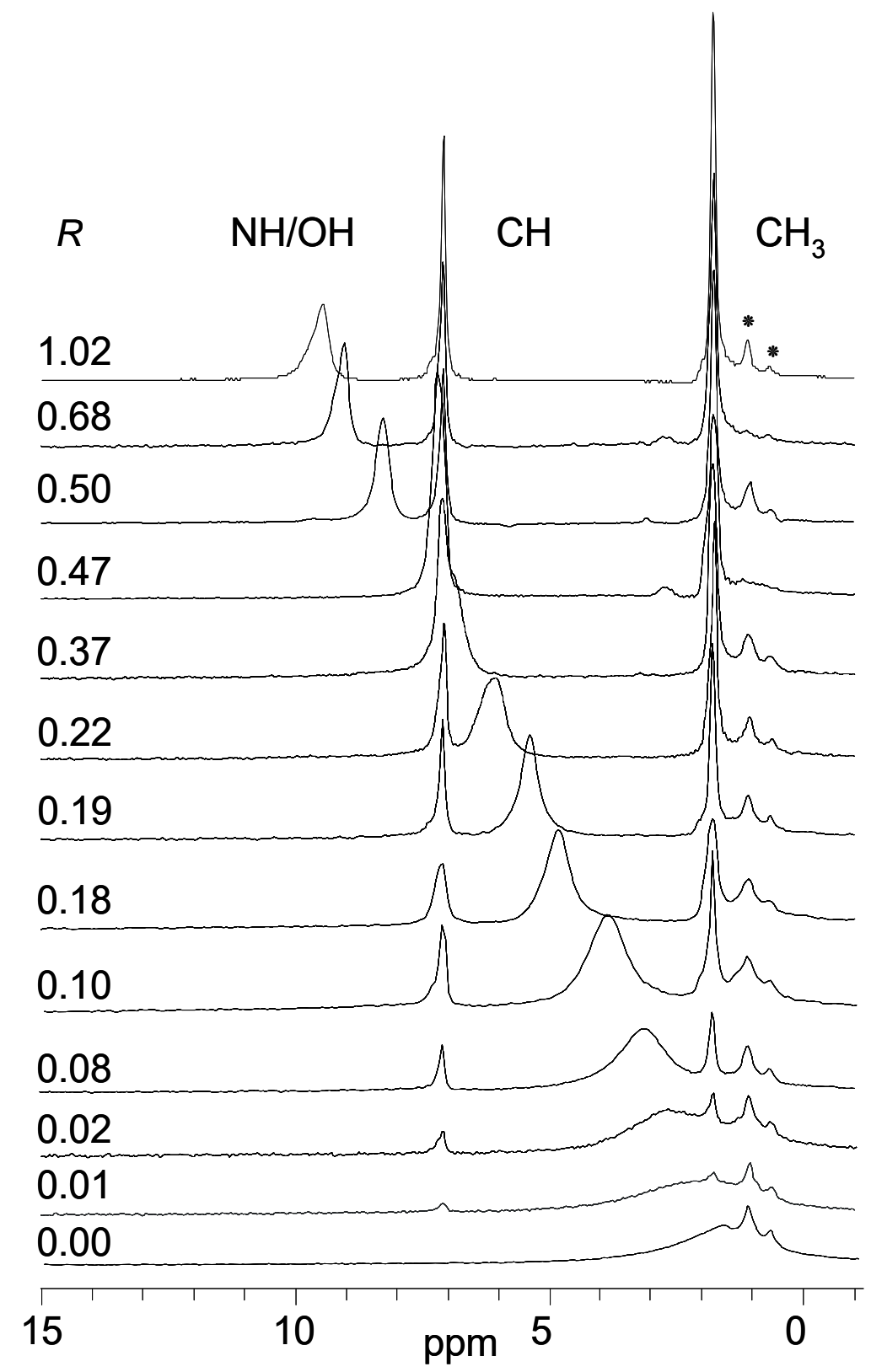


Figure S2. Selected single $90^{\circ}$ pulse ${ }^{1} \mathrm{H}$ MAS NMR spectra at $398 \mathrm{~K}$ and $7 \mathrm{~T}$ of 4-methyl- $1 H$ pyrazole in SBA-15.

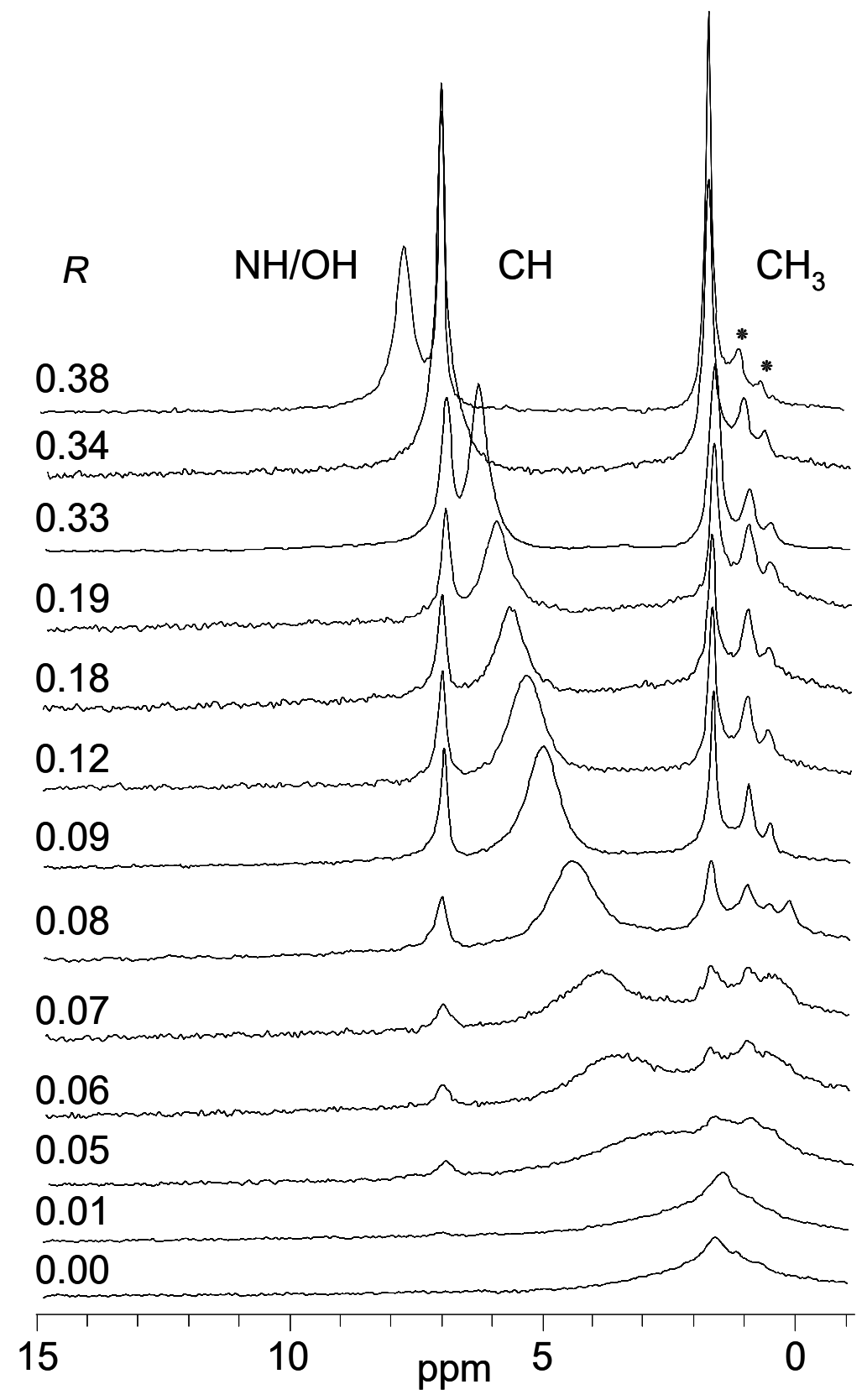


- S6 -

Figure S3. Density of neat 4-methyl-pyrazole at different temperatures according to the Data of Table 3.

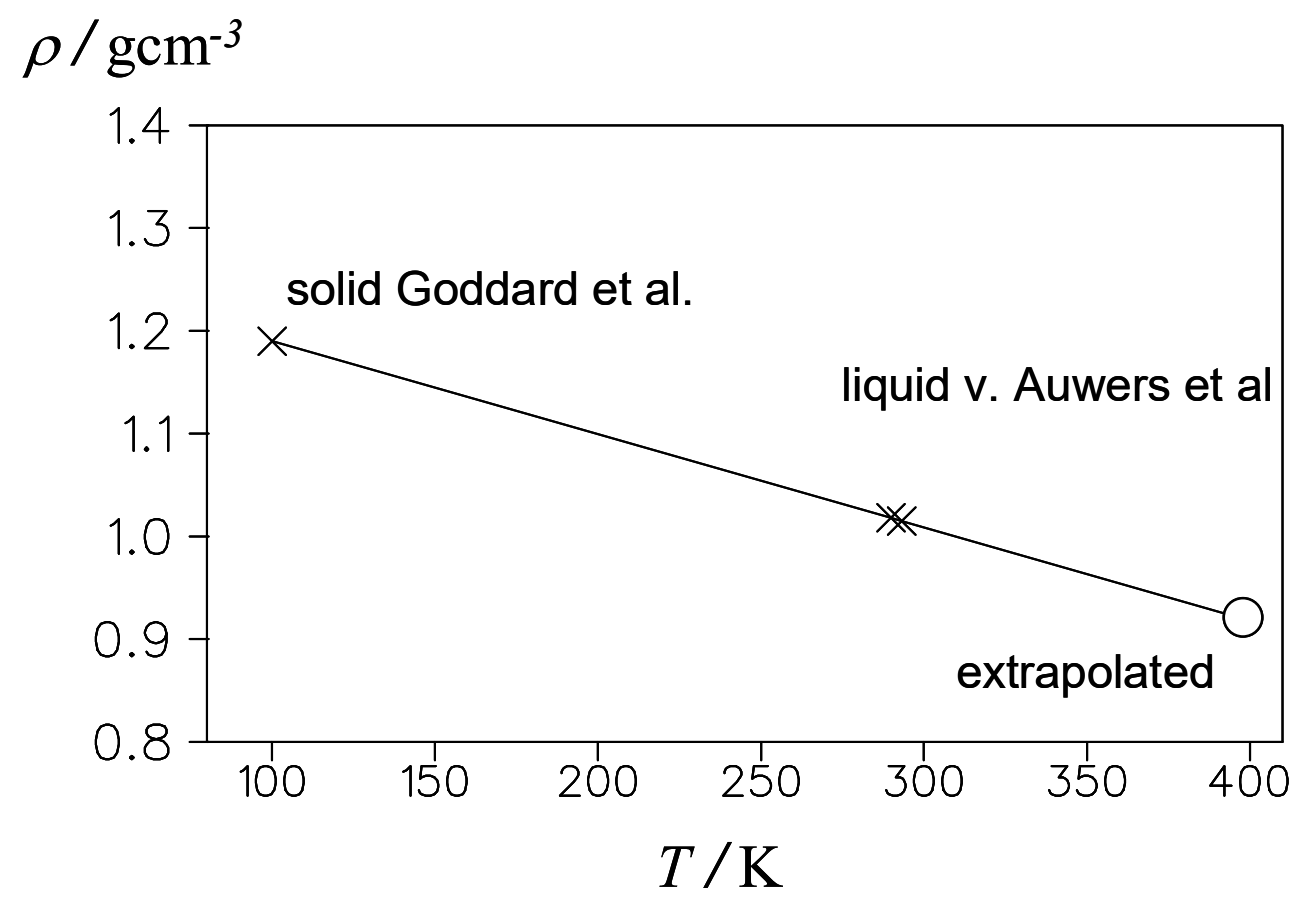

